\title{
Effect of topological defects on graphene geometry and stability
}

This article was published in the following Dove Press journal:

Nanotechnology, Science and Applications

7 October 2010

Number of times this article has been viewed

\section{Ibolya Zsoldos}

Faculty of Technology Sciences, Szechenyi Istvan University, Gyor, Hungary
Correspondence: Ibolya Zsoldos Faculty of Technology Sciences, Szechenyi Istvan University, Egyetem t. I, H-9026 Gyor, Hungary Email zsoldos.ibolya@gek.szie.hu
Abstract: The effect of two basic topological defects, mitosis and the Stone-Wales defect, is studied in the graphene structure. The topological rules of the curvatures due to the occurrence of the defects in different arrangements are determined. Despite the fact that the causes and the probability of these topological defects are not known today, this theoretical work studies the distortions caused by the defects geometry and stability of the graphene structure.

Keywords: graphene structure, topological rules, nanotube, Stone-Wales defect

\section{Introduction}

It is known that pentagons built between hexagons cause the curvatures in the structures of the fullerenes and the carbon nanotube ends. ${ }^{1,2}$ The characteristic saddle-shaped surfaces at the junctions of the carbon nanotubes result in the heptagons built in the hexagonal structure. ${ }^{3,4}$ In this work the deforming effect of the pentagons and the heptagons as defects of the graphene structure is studied.

Pentagons and heptagons generally can appear in pairs in the graphene structure since the mean coordination number of the planar trivalent polygonal cell systems equals 6 according to Euler's law.,

The effect of the Stone-Wales defect was studied on the mechanical, ${ }^{7-9}$ fracture, ${ }^{10}$ and electronic ${ }^{11-13}$ properties of single-wall carbon nanotubes by different theoretical methods. In the case of the graphene structure it was observed that the Stone-Wales defect can modify the structure from planar to spatial. ${ }^{15}$ The Raman spectra was calculated ${ }^{14}$ and the electronic behavior was estimated. ${ }^{17}$

In this work the geometric effect of topological defects is examined to answer the question: does the graphene structure containing defects remain planar or not?

\section{Method}

Considering the topological knowledge of the planar cell systems, the two characteristic examples of their origin are:

- The Stone-Wales defect is the $90^{\circ}$ rotation of two carbon atoms with respect to the midpoint of the bond. In the Stone-Wales defect, four hexagons are changed into two pentagons and two heptagons (Figure 1a). The pentagons are separated by heptagons. The number of the atoms (vertexes) does not change.

- The mitosis where two pentagons originate from a given hexagon and consequently the neighboring hexagons become heptagons (Figure 1b). The heptagons are separated by pentagons. The number of the atoms increases by two. 


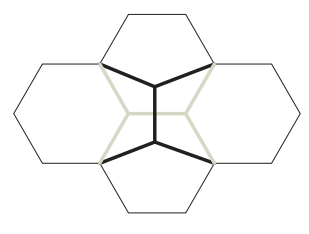

a

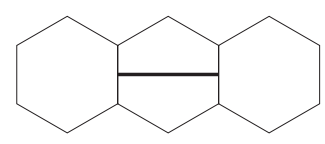

b

Figure I a) The Stone-Wales defect: the gray heavy lines show the bonds before the transformation and the black heavy lines show the bonds after the transformation. b) Mitosis defect: The black heavy line show the new bond between the new atoms.

Considering the possibilities for the appearing of the topological defects in the graphene structure the following arrangements can be studied:

- the defect itself,

- defects arranged along lines,

- defects arranged in groups,

- defects arranged in net.

An example for every possibility was studied in this work. A relaxation procedure was applied for the different arrangements of the topological defects. The inputs were planar patterns where the defects were constructed in the graphene structure similarly as is shown in Figure 1. The goal of the relaxation procedure was to find the equilibrium state of the structure applying an energetic potential function, which is a mathematical formula for the energy of the chemical bonds. The procedure is a simplified variation of the molecular dynamic. ${ }^{18,19}$ The equilibrium state was reached by an iteration procedure varying the coordinates of the atoms (for example with the known mathematical gradient method) until the minimum of the energetic potential function is found. For this purpose two methods were tried out in parallel: the Desktop Molecular Modeller, which is a commercial software, and our own computer codes based on the energetic Brenner empirical potential function. ${ }^{20}$ Both methods have given the same result for all examples shown in this work.

In every model shown in the following sections, an infinite structure is assumed with a periodic boundary condition.

\section{Results and discussion The effect of the Stone-Wales defects}

The pentagon pair separated by the heptagon pair as the result of the Stone-Wales defect occurring in the graphene structure was studied first. According to the relaxation procedures, the arrangement containing one defect remained planar (Figure 2). Both the bond lengths and the angles became distorted. The rates of the distortions are summarized in Table 1. The percentile rates were computed for the

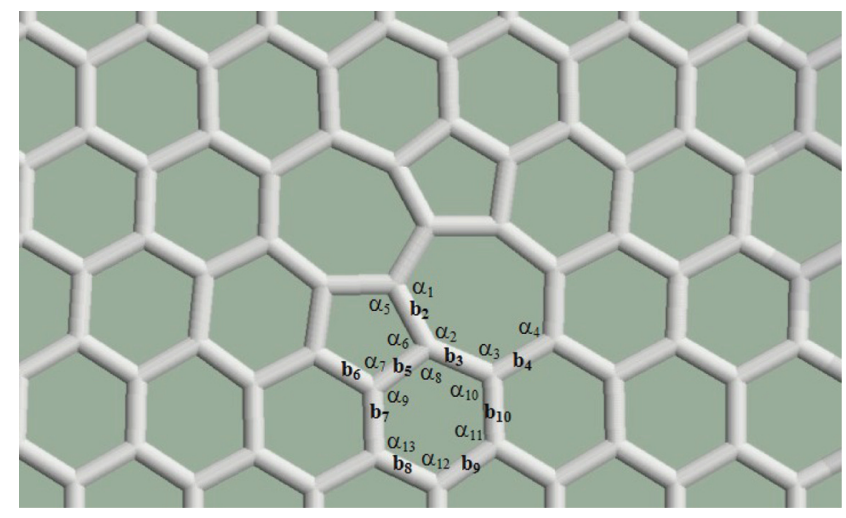

Figure 2 The graphene structure containing a Stone-Wales defect remains planar.

data of regular pentagons, hexagons, and heptagons, for the bond length of the perfect graphene (1.42 Angström), and for the angles of the regular polygons $\left(108^{\circ}\right.$ in the case of the regular pentagons, $120^{\circ}$ in the case of the regular hexagons, and $128.57^{\circ}$ in the case of the regular heptagons). Smaller deviations were found in the bond lengths (the largest deviation is $2.87 \%$ at $b_{1}$ ) and larger deviations were found in the angles (the largest deviation is $10.6 \%: \alpha_{2}$ inside heptagons).

Several further examples were studied for the cases when the structure contains more defects. Pentagon-heptagon pairs are arranged in a line as shown in Figure 3. The orientation of the crystal structure of the graphene is above the defect line (zigzag) rather than under the defect line (armchair). The structure remains planar according to the relaxation procedures.

Table I The deformation rates for the structure shown in Figure 2

\begin{tabular}{|c|c|c|c|c|}
\hline \multicolumn{2}{|l|}{ Bond lengths } & \multicolumn{3}{|l|}{ Angles } \\
\hline $\begin{array}{l}\text { Notations in } \\
\text { Figure } 7\end{array}$ & $\begin{array}{l}\text { Rate of } \\
\text { distortion } \\
(\%)\end{array}$ & $\begin{array}{l}\text { Notations in } \\
\text { Figure } 7\end{array}$ & Angle & $\begin{array}{l}\text { Rate of } \\
\text { distortion } \\
(\%)\end{array}$ \\
\hline$b_{1}$ & 97.14 & $\alpha_{1}$ & 120.90 & 94.03 \\
\hline$b_{2}$ & $100.7 \mid$ & $\alpha_{2}$ & 142.20 & 110.60 \\
\hline$b_{3}$ & $100.7 \mid$ & $\alpha_{3}$ & 125.98 & 97.98 \\
\hline $\mathrm{b}_{4}$ & $100.7 \mid$ & $\alpha_{4}$ & 121.84 & 94.77 \\
\hline $\mathrm{b}_{5}$ & 97.86 & $\alpha_{5}$ & 118.22 & 109.46 \\
\hline $\mathrm{b}_{6}$ & 97.86 & $\alpha_{6}$ & 98.56 & 91.26 \\
\hline$b_{7}$ & 97.86 & $\alpha_{7}$ & 112.33 & 104.01 \\
\hline$b_{8}$ & 98.57 & $\alpha_{8}$ & 119.24 & 99.37 \\
\hline$b_{9}$ & 100.00 & $\alpha_{9}$ & 125.28 & 104.40 \\
\hline \multirow[t]{4}{*}{$b_{10}$} & 101.42 & $\alpha_{10}$ & 116.05 & 96.71 \\
\hline & & $\alpha_{11}$ & 122.29 & 101.91 \\
\hline & & $\alpha_{12}$ & 120.04 & 100.03 \\
\hline & & $\alpha_{13}$ & 117.10 & 97.58 \\
\hline
\end{tabular}




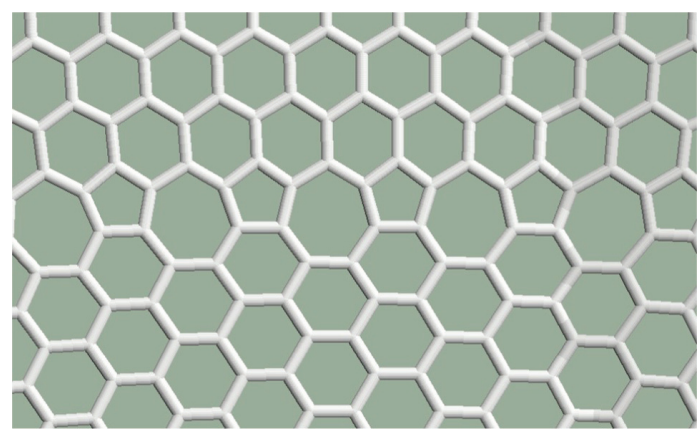

Figure 3 The defects are arranged in a horizontal line.

In Figure 4, perfect hexagonal and pentagon-heptagon chains succeed each other. Half of the cells are not hexagonal. The structure remains planar.

In Figure 5 the pentagon-heptagon pairs are arranged in a net. The nearest pentagons are separated by heptagons (or a heptagon and a hexagon) everywhere in the structure. Two-thirds of the cells are not hexagonal. The structure remains planar.

According to the relaxation procedures applied in this theoretical work, the graphene structure containing Stone-Wales defects remain planar. This should be related with the fact that this defect can be seen as a dislocation dipole. It seems that there is a cancellation in the distortions originated by the dislocations. ${ }^{21}$

\section{The effect of mitosis as topological defect}

Mitosis as topological defect was studied in trivalent polygonal cell systems ${ }^{6}$ and was not studied in the graphene structure till now. This section is a theoretical consideration to determine the effect of the presence of such topological defects on the geometrical structure of the graphene.

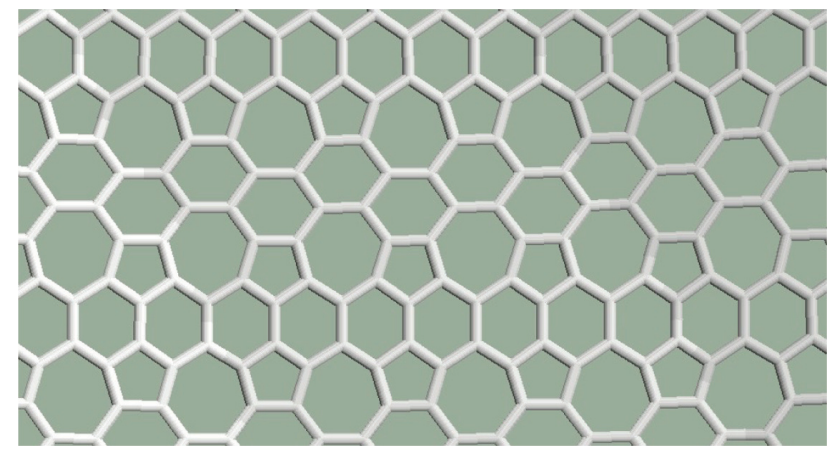

Figure 4 Horizontal defect lines consisting of pentagon-heptagon pairs are constructed between the hexagonal lines.

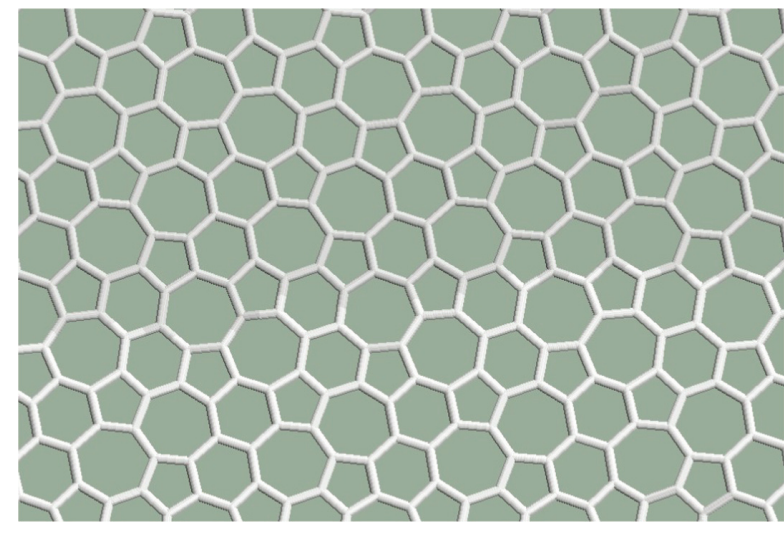

Figure 5 The pentagon-heptagon pairs are arranged in a net.

In a first approximation, the case of mitosis as topological defect is very interesting since the relaxation procedures give a planar structure if the heptagon pair separated by the pentagon pair is studied alone (Figure 6), but the structure is not planar if this defect is constrained in the graphene structure (Figure 7).

Both the bond lengths and the angles become distorted in the spatial structure. The rates of the distortions are summarized in Table 2. The percentile rates are computed to the bond length of the perfect graphene and to the angles of the regular polygons. It is true here again that smaller deviations were found in the bond lengths and larger deviations were found in the angles, the order of magnitude of the rates is similar to the case of the Stone-Wales defect.

Figure 8 shows mitoses arranged next to each other along a line. The deformations are summarized along the line, the sum is very large, and the solution is a wavy pattern with alternating curvatures.

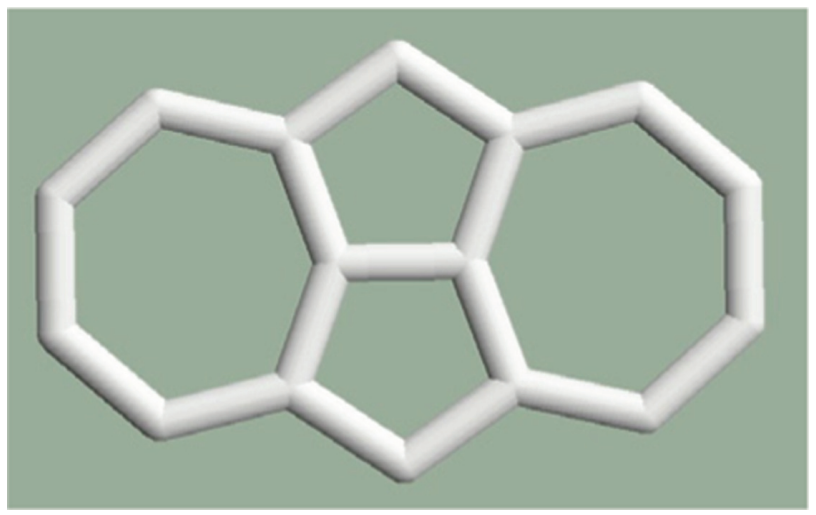

Figure 6 The structure of the heptagon pair separated by the pentagon pair is planar if it is studied alone. 

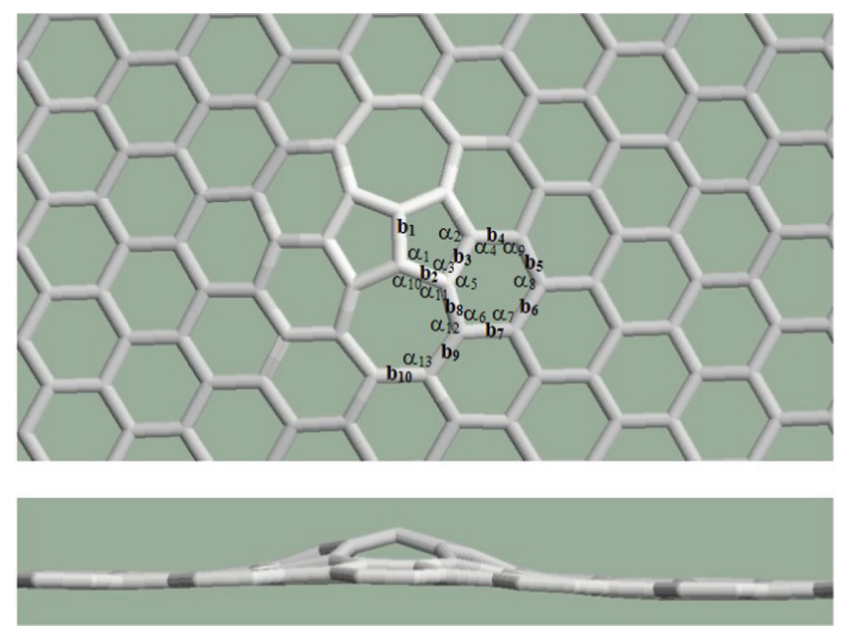

Figure 7 A curvature appears in the structure containing mitosis as topological defect. Upper: top view, lower: side view.

Mitoses can be arranged next to each other not only along straight lines but along curves or in groups. For example: three pentagons placed next to each other produce a larger curvature in the graphene structure than in the case of two pentagons (Figure 9). It is interesting that the largest curvature arises from six mitoses arranged in a group (Figure 10). In this case, six pentagons placed next to each other created a half dodecahedron, which can be the end of an armchair-type nanotube. If the six pentagons are arranged along a curve as can be seen in Figure 11, the resulting structure is the end of a zigzag-type nanotube. To arrange more than six pentagons next to each other cannot be solved in a pentagon-heptagon-hexagon system.

A significant difference between the two basic types of topological defects is that the pentagons are next to each other

Table 2 The deformation rates of bond lengths and the angles

\begin{tabular}{|c|c|c|c|c|}
\hline \multicolumn{2}{|l|}{ Bond lengths } & \multicolumn{3}{|l|}{ Angles } \\
\hline $\begin{array}{l}\text { Notations in } \\
\text { Figure } 2\end{array}$ & $\begin{array}{l}\text { Rate of } \\
\text { distortion } \\
\text { (\%) }\end{array}$ & $\begin{array}{l}\text { Notations in } \\
\text { Figure } 2\end{array}$ & Angle & $\begin{array}{l}\text { Rate of } \\
\text { distortion } \\
(\%)\end{array}$ \\
\hline$b_{1}$ & 98.57 & $\alpha_{1}$ & 111.62 & 103.35 \\
\hline$b_{2}$ & 97.14 & $\alpha_{2}$ & 118.04 & 109.29 \\
\hline$b_{3}$ & 98.57 & $\alpha_{3}$ & 99.32 & 91.96 \\
\hline $\mathrm{b}_{4}$ & 96.43 & $\alpha_{4}$ & 120.60 & 100.5 \\
\hline$b_{5}$ & 99.29 & $\alpha_{5}$ & 128.72 & 107.27 \\
\hline$b_{6}$ & $100.7 \mid$ & $\alpha_{6}$ & 107.52 & 89.6 \\
\hline$b_{7}$ & 100.00 & $\alpha_{7}$ & 123.68 & 103.67 \\
\hline $\mathrm{b}_{8}$ & 98.57 & $\alpha_{8}$ & 122.86 & 102.38 \\
\hline$b_{9}$ & 100.43 & $\alpha_{9}$ & 113.72 & 94.77 \\
\hline \multirow[t]{4}{*}{$b_{10}$} & 100.70 & $\alpha_{10}$ & 126.40 & 98.31 \\
\hline & & $\alpha_{11}$ & 130.46 & 101.47 \\
\hline & & $\alpha_{12}$ & 125.80 & 97.85 \\
\hline & & $\alpha_{13}$ & 127.64 & 99.28 \\
\hline
\end{tabular}
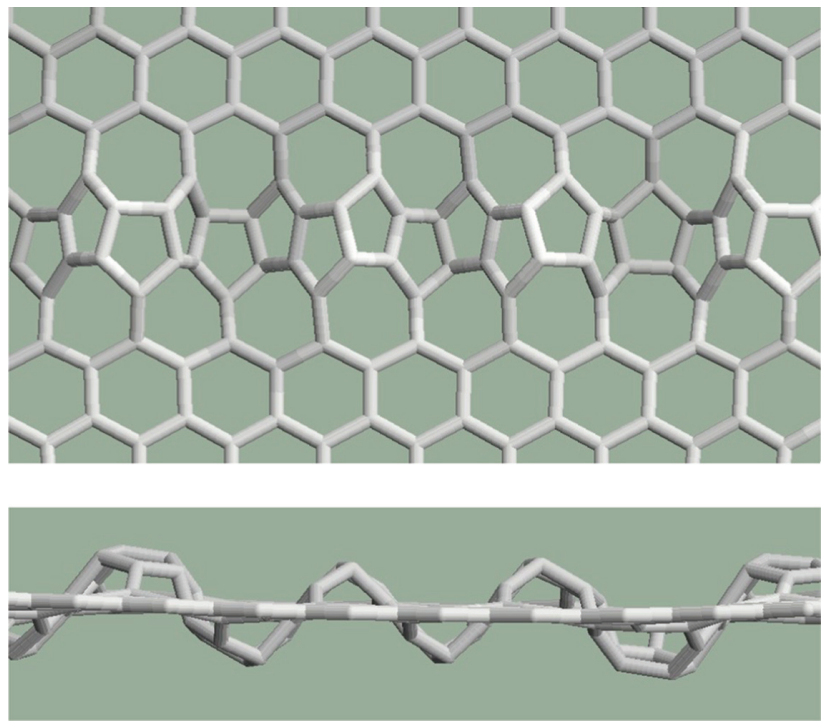

Figure 8 Mitoses arranged along a straight line. Upper: top view, lower: side view.

at the mitosis, separated in a Stone-Wales defect, and according to the presented examples that there are curvatures in the graphene structure when pentagons are placed next to each other. However, pentagons can be placed next to each other if two Stone-Wales defects are created fittingly, as shown in Figure 12.

Finally do pentagons and/or heptagons occur in the system alone (such patterns can be created using Stone-Wales defect series)? When a pentagon is surrounded by hexagons, a spherical surface forms and when a heptagon is surrounded by hexagons, the characteristic saddle-shaped surface forms. The example shown in Figure 13 contains several pentagons and
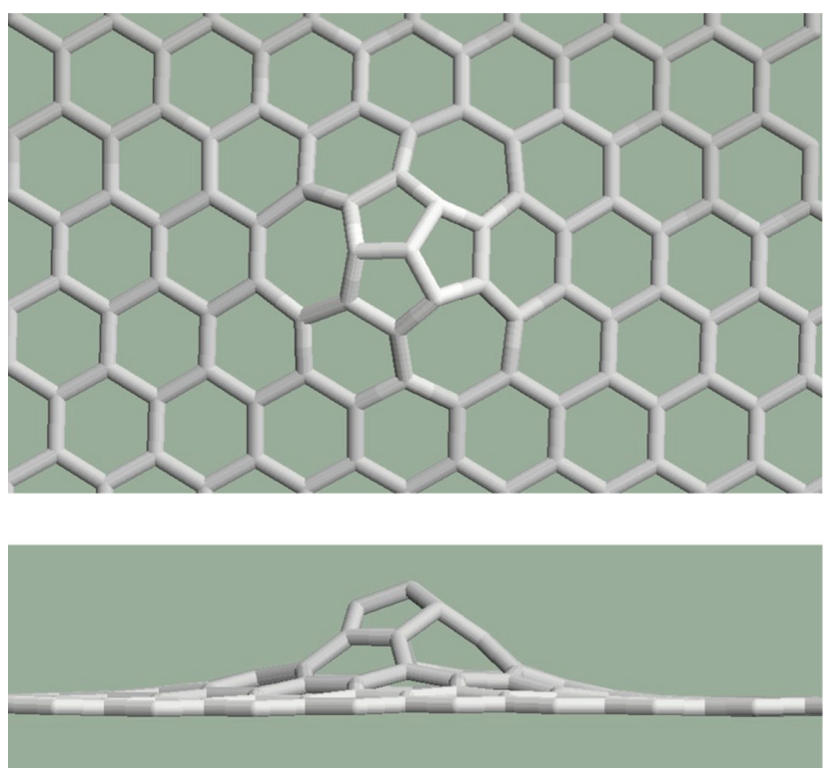

Figure 9 Three pentagons are constructed next to each other. Upper: top view, lower: side view. 

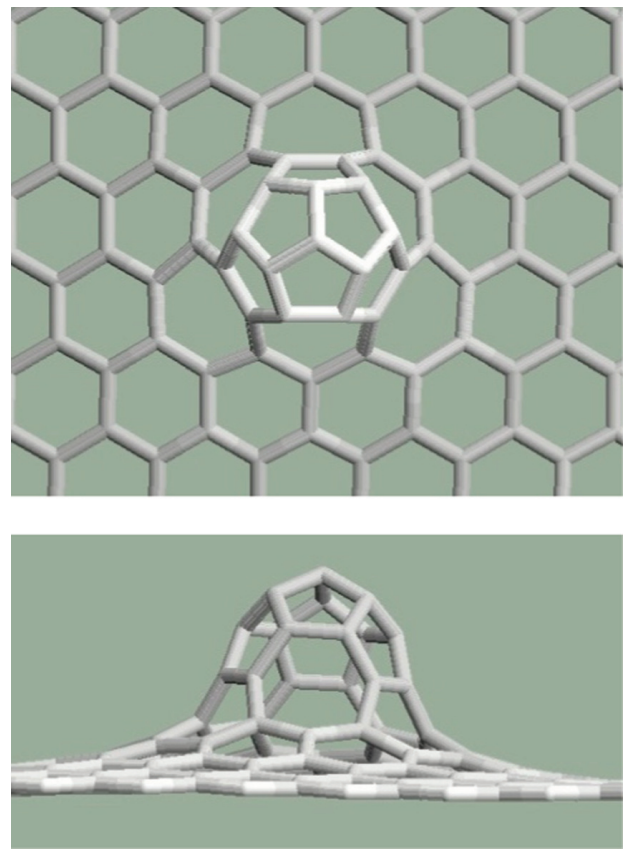

Figure 10 Six pentagons are placed next to each other in a group. Upper: top view, lower: side view.

heptagons, in a hexagonal environment. Relaxation procedures create a surface with alternate curvatures for this pattern.

\section{The stability in the environment of the defects}

To study the stability of the distorted graphene structures, the cohesive energy (the average energy of the chemical
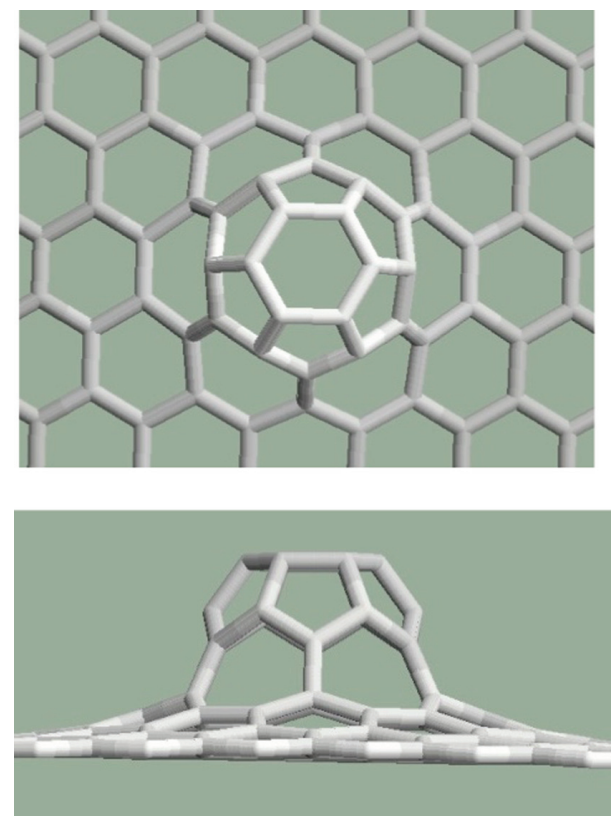

Figure II Six pentagons are placed next to each other along a curve. Upper: top view, lower: side view.
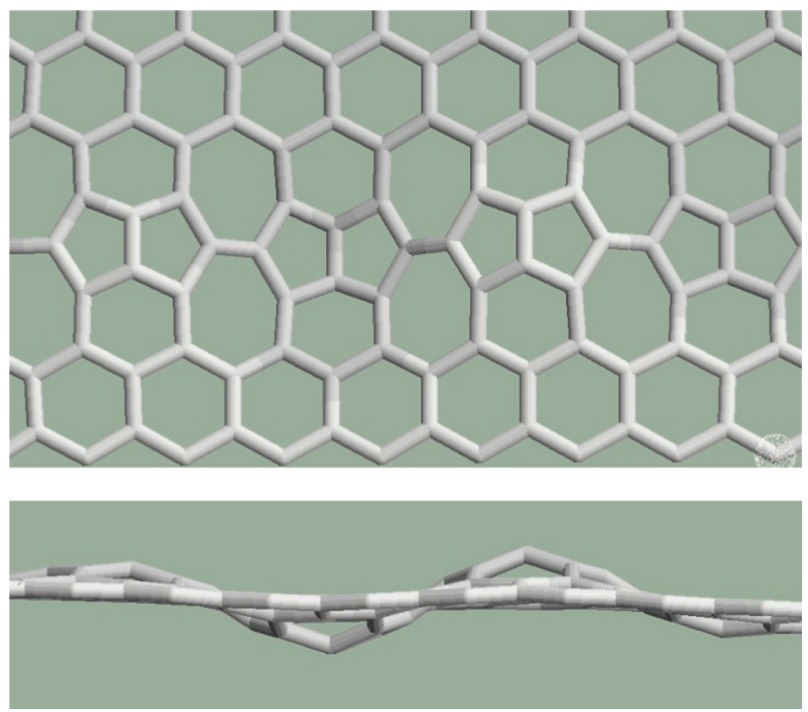

Figure 12 Stone-Wales defects placed next to each other at the pentagons cause curvature Upper: Top view, lower: side view.

bonds: the total energy divided by the number of the bonds) was calculated for the structures shown above. The Brenner empirical potential function was used for this purpose and averages were calculated only for the closest environments of the defects (for the bonds connecting with pentagons or heptagons), this better emphasizes the effect of the defects. Cohesive energy values are summarized in Table 3.

Cohesive energy in the environment of the defects increases several percent compared to the cohesive energy of the perfect graphene $(-7.44 \mathrm{eV})$ in every case. The increase is smaller for the planar structures (Figures 3-6) and it is larger for the structures with curvatures (Figures 8-12). The more pentagons are connected to each other, the larger the decrease of stability (Figures 8, 10-12). The defects shown in Figure 8 have the least stability because the pentagons

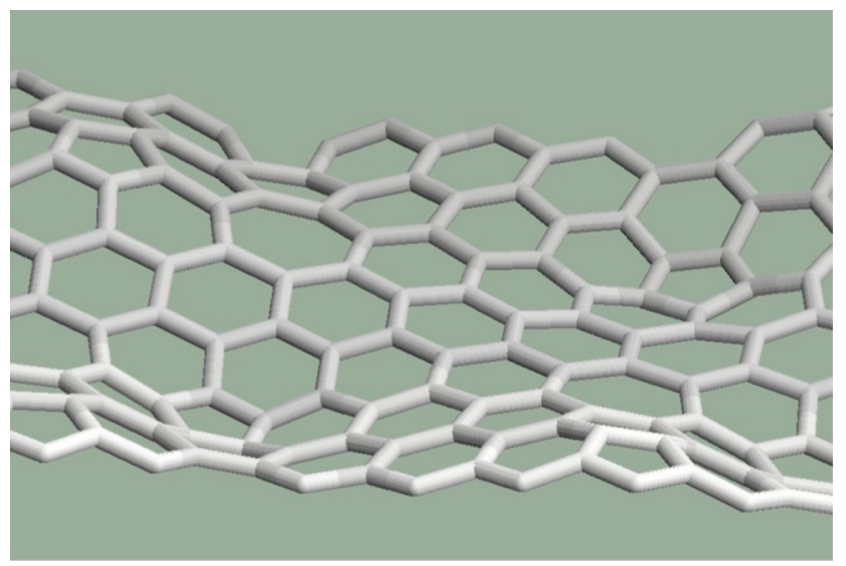

Figure I 3 Pentagons and heptagons surrounded by hexagons in the graphene structure. 
Table 3 The cohesive energy for the environment of the defects

\begin{tabular}{ll}
\hline Structure & Ec $(\mathrm{eV})$ \\
\hline Figure 2 & -7.18 \\
Figure 3 & -7.17 \\
Figure 4 & -7.16 \\
Figure 5 & -7.19 \\
Figure 7 & -7.12 \\
Figure 8 & -6.86 \\
Figure 9 & -7.09 \\
Figure 10 & -7.01 \\
Figure II & -7.03 \\
Figure 12 & -7.03 \\
Figure 13 & -7.14 \\
\hline
\end{tabular}

cause curvatures in both sides of the graphene sheet where they are connected with each other.

\section{Conclusions}

After considering the theoretical work of this study, shown in this work the following topological rules can be formulated. The graphene structure containing pentagonal and heptagonal defects does not remain planar if:

- at least two pentagons can be found next to each other, or

- all neighbor cells of a pentagon or a heptagon are hexagons.

With a more general topological description, curvatures can be found where:

- the structure contains pentagons where the sum of the edge numbers of the nearest neighbor polygons is $\leq 31$, or

- it contains heptagons where the sum of the edge numbers of the nearest neighbor polygons is $\geq 42$.

In the environment of the studied topological defects, the stability of the structure decreases by (the cohesive energy increases) several percent.

\section{Acknowledgment}

This work was supported by OTKA grants K 73776 and TÁMOP grants TÁMOP-4.2.1/B-09/1/KONV-2010-2013 in Hungary.

\section{Disclosure}

The author reports no conflicts of interest in relation to this work.

Nanotechnology, Science and Applications

\section{Publish your work in this journal}

Nanotechnology, Science and Applications is an international, peerreviewed, open access journal that focuses on the science of nanotechnology in a wide range of industrial and academic applications. It is characterized by the rapid reporting across all sectors, including engineering, optics, bio-medicine, cosmetics, textiles, resource sustainability

\section{References}

1. Itoh S, Ordejon P, Drabold DA, et al. Structure and energetics of giant fullerenes: An order-N molecular-dynamics study. Phys Rev B. 1996;53(4):2132-2140.

2. Lordi V, Ma SXC, Yao N. Towards probing pentagons on carbon nanotube tips. Surf Sci. 1999;421(1-2):L150-L155.

3. Biro LP, Ehlich R, Osvath Z, et al. From straight carbon nanotubes to Y-branched and coiled carbon nanotubes. Diamond Relat Mater. 2002;11(3-6):1081-5108.

4. Lisenkov SV, Ponomareva IV, Chernozatonskii LA. Basic configuration of a single-wall carbon nanotube Y junction of D-3h symmetry: Structure and classification. Physics of the Solid State. 2004;46:(8):1577-1582.

5. Weaire D, Rivier N. Soap, cells and statistics-random patterns in two dimensions. Contemp Phys. 1984;25(1):55-99.

6. Mombach JCM, de Almeida RMC, Iglesias JR. Mitosis and growth in biological tissues. Phys Rev E Stat Phys Plasmas Fluids Relat Interdiscip Topics. 1993;48(1):598-602.

7. Pozrikidis C. Effect of the Stone-Wales defect on the structure and mechanical properties of single-wall carbon nanotubes in axial stretch and twist. Arch Appl Mech. 2009;79(2):113-123.

8. Nasdala L, Ernst G, Lengnick M, Rothert H. Finite element analysis of carbon nanotubes with Stone-Wales defects. Comput Model Eng Sci. 2005;7(3):293-304.

9. Lu Q, Bhattacharya B. Effect of randomly occurring Stone-Wales defects on mechanical properties of carbon nanotubes using atomistic simulation. Nanotechnology. 2005;16(4):555-566.

10. Tserpes KI, Papanikos P. The effect of Stone-Wales defect on the tensile behavior and fracture of single-walled carbon nanotubes. Composite Structures. 2007;79(4):581-589.

11. Fang X, Hu HF, Wei JW, Zeng H. Peng P. [Effects of topological defects on the electronic structure and optical spectrum of single-wall carbon nanotubes.] Guang Pu Xue Yu Guang Pu Fen Xi. 2007;27(7):1267-1270.

12. Eom JH, Lee H, Im J, et al. Electronic structure of defects and quantum transport in carbon nanotubes. Physica B: Condensed Matter. 2006; 376-377:7-10.

13. Pachos JK. Manifestation of topological effects in graphene. Cont Phys. 2009;50:375-389

14. Hatsugai Y, Fukui T, Aoki H. Topological aspects of graphene. Eur Phys J Special Topics. 2007;148(1):133-141.

15. Ma J,Alfe D, Michaelides A, Wang E. Stone-Wales defects in graphene and other planar sp(2)-bonded materials. Phys Rev B. 2009;80(3):033407.

16. Popov VN, Henrard L, Lambin P. Resonant Raman spectra of graphene with point defects. Carbon. 2009;47(10):2448-2455.

17. Amara H, Latil S, Meunier V, Lambin P, Charlier J-C. Scanning tunneling microscopy fingerprints of point defects in graphene: A theoretical prediction. Phys Rev B. 2007;76(11):115423.

18. Terrones M, Banhart F, Grobert N, Charlier J-C, Terrones H, Ajayan PM. Molecular junctions by joining single-walled carbon nanotubes. Phys Rev Lett. 2002;89(7):075505-1.

19. Ponomareva I, Chernozatonskii LA, Andriotis AN, Menon M. Formation pathways for single-wall carbon nanotube multiterminal junctions. New J Phys. 2003;5:119.1-119.12.

20. Brenner DW. Empirical potential for hydrocarbons for use in simulating the chemical vapor deposition of diamond films. Phys Rev B. 1990; 42(15):9458-9471.

21. Seung HS, Nelson DR. Defects in flexible membranes with crystalline order. Phys Rev A. 1988;38(2):1005-1018.

\section{Dovepress}

and science. Applied research into nano-materials, particles, nanostructures and fabrication, diagnostics and analytics, drug delivery and toxicology constitute the primary direction of the journal. The manuscript management system is completely online and includes a very quick and fair peer-review system, which is all easy to use. 